\title{
Robotic repair of vesicovaginal fistula - initial experience
}

\author{
Ankush Jairath ${ }^{1}$, Sudharsan S.B ${ }^{1}$, Shashikant Mishra ${ }^{1}$, Arvind Ganpule ${ }^{1}$, Ravindra Sabnis ${ }^{1}$, Mahesh \\ Desai $^{1}$
}

${ }^{1}$ Department of Urology, Muljibhai Patel Urological Hospital (MPUH). Nadiad, India

\section{ABSTRACT}

Objective: The most common acquired fistula of the urinary tract is Vesicovaginal fistulae (VVF) (1) posing social stigmata for the patient as well as a surgical challenge for the urologist. Here we present our initial experience with Robotic assisted laparoscopic repair of VVF, its safety and efficacy.

Materials and Methods: Seven out of eight fistulas were post hysterectomy; five had undergone abdominal while two had laparoscopic hysterectomy while one was due to prolonged labour. Two had associated ureteric injury. All underwent robotic assisted laparoscopic trans abdominal extravesical approach. Three $8 \mathrm{~mm}$ ports for robotic arms, one $12 \mathrm{~mm}$ port for camera and another $12 \mathrm{~mm}$ for assistant were used in a fan shaped manner. All had preoperative ureteric catheter placed. Bladder was closed in two layers and vagina in one layer. Omental flap placed in all cases except two where it was not possible. Drain and per urethral catheter placed in all cases. Double J stents were placed in two cases requiring ureteric implantation additionally.

Results: The mean age of presentation was 39.25 years (26-47 range) with mean BMI being $26.25 \mathrm{~kg} / \mathrm{m} 2$ (21-32 range). Mean duration between insult and repair was 9.37 months (3-24 months). Only in single case there was history of previous repair attempt. On cystoscopy four had supratrigonal VVF and four were trigonal with mean size of $13.37 \mathrm{~mm}$ (7-20 mm). Mean operative time was 117.5 minutes (90-150). There were no intraoperative/postoperative complications or need for open conversion. Mean haemoglobin drop was $1.4 \mathrm{gm} / \mathrm{dL}$ (0.3-2 gm). Drain was removed once 24-48 hours output is negligible. One patient had post-operative urinary leak at 2 weeks which ceased with continuation of catheterisation for another 2 weeks. Catheter was removed after voiding cystourethrogram showed no leak at 2-3 weeks postoperatively. Mean duration of drain was 3.75 days (3-5) and per urethral catheterisation (which was removed after voiding cystourethrography) was 15.75 days (9-28). Mean hospital stay was 6.62 days (4-14). Post-operative bladder capacity was 324.28 cc (280-350) on voiding diary. Follow up ranged from 3-9 months. At 3 months of follow-up, these patients continued to void normally and there was no evidence of recurrence of VVF.

Conclusion: Robotic repair of VVF is safe and feasible and has additional advantages in the form of precise suturing under 3D vision and certainly a more striking and effective option especially in complex VVF repair associated with ureteric injuries (2).

\section{REFERENCES}

1. Gerber GS, Schoenberg HW. Female urinary tract fistulas. J Urol. 1993;149:229-36.

2. Sundaram BM, Kalidasan G, Hemal AK. Robotic repair of vesicovaginal fistula: case series of five patients. Urology. 2006;67:970-3.

\section{ARTICLE INFO}

Available at: http://www.intbrazjurol.com.br/video-section/video-library/jairath_168_169/

Int Braz J Urol. 2016; 42 (Video \#1): 168-9 
Submitted for publication:

March 05, 2015

Accepted after revision:

July 25, 2015
Correspondence address:

Ankush Jairath, MD

Department of Urology

Muljibhai Patel Urological Hospital

(MPUH)

Nadiad, India

Telephone: + 91268 252-0323

E-mail: ankushjairath@gmail.com

\section{EDITORIAL COMMENT}

These authors have reported one of the largest series of robotic assisted laparoscopic repair of vesico-vaginal fistula (VVF). They have demonstrated that robotic repair of VVF is associated with minimal morbidity with excellent 3 month follow up outcomes. As urologists have advanced their robotic expertise, they are now able to tackle the more difficult cases of pelvic reconstruction. VVF can represent a challenge due to both etiology and variability in anatomy. The authors are correct in their commentary that the robotic platform with its $3 \mathrm{D}$ vision system and precise instrumentation in the pelvis allow it to be an effective option when considering VVF repair. While urologists can add this to their robotic armamentarium, I would caution that such cases should be only attempted by seasoned robotic surgeons.

Trushar Patel, MD Assistant Professor of Urology Director of Robotic Urologic Surgery, Florida Hospital Tampa Morsani College of Medicine University of South Florida, USA E-mail: trushar.patelmd@gmail.com 